\title{
Momentum-space calculation of proton-deuteron scattering including Coulomb and irreducible three-nucleon forces
}

\author{
A. Deltuva* \\ Centro de Física Nuclear da Universidade de Lisboa, P-1649-003 Lisboa, Portugal
}

(Received October 26, 2009)

\begin{abstract}
Three-nucleon scattering equations with irreducible three-nucleon force are solved in momentumspace. The Coulomb interaction between the two protons is included using the method of screening and renormalization. The need for the renormalization of the scattering amplitudes is demonstrated numerically. The Coulomb and Urbana IX three-nucleon force effects on the observables of elastic proton-deuteron scattering and breakup are studied.

PACS numbers: 21.30.-x, 21.45.-v, 24.70.+s, 25.10.+s
\end{abstract}

\section{INTRODUCTION}

Nucleon-deuteron scattering has been extensively studied in the past with the aim of testing various nuclear interaction models, i.e., nucleon-nucleon $(N N)$ potentials and also three-nucleon $(3 N)$ forces. While the theoretical description of neutron-deuteron $(n d)$ scattering is considerably simpler and a number of highly sophisticated calculations [1 [5] exists with realistic $N N$ potentials and $3 N$ forces, the experimental data are much more abundant and precise for proton-deuteron $(p d)$ reactions. In that case there are only few realistic calculations above the deuteron breakup threshold. Configuration-space treatment based on the Kohn variational principle [6, 7] uses the local Argonne $V_{18}$ (AV18) $N N$ potential [8] and the irreducible Urbana IX (UIX) $3 N$ force [9] but is limited so far to elastic scattering at energies below $50 \mathrm{MeV}$ in the center-of-mass (c.m.) system; only very recent work [10] solving integral Faddeev equations in configuration-space provides $p d$ breakup results at proton lab energy $13 \mathrm{MeV}$. In contrast, the momentum-space treatment [11, 12] based on the screening and renormalization method [13, 14] has lead to $p d$ elastic scattering and breakup results for energies up to pion-production threshold; this method can be used with nonlocal $N N$ potentials as well, but so far only an effective $3 N$ force due to the virtual excitation of a nucleon to a $\Delta$ isobar [3] has been included. The aim of the present work is to overcome that limitation, i.e., to extend the technique of Refs. 11, 12] to include also an irreducible $3 N$ force.

In Sec. [II we derive three-particle scattering equations including the three-body force. In Sec. III we discuss the inclusion of the Coulomb interaction using the method of screening and renormalization and demonstrate its validity numerically. Section [V] presents some characteristic effects of Coulomb and Urbana IX $3 N$ force in $p d$ elastic scattering and breakup. Section $\nabla$ gives our summary.

*deltuva@cii.fc.ul.pt

\section{THREE-PARTICLE SCATTERING EQUATIONS INCLUDING IRREDUCIBLE THREE-BODY FORCE}

For the description of three-particle scattering interacting via three pairwise potentials $v_{\alpha}, \alpha=1,2,3$, we used Alt, Grassberger, and Sandhas (AGS) equations [15]. In this section we give a short derivation of the AGS equations including a three-body force

$$
V_{(3)}=\sum_{\alpha=1}^{3} u_{\alpha}
$$

which is decomposed into three terms $u_{\alpha}$ that are symmetric in the exchange of particles $\beta \neq \alpha$ and $\gamma \neq \alpha$ and can be transformed into one another by a cyclic permutation; these properties will be used later for the symmetrization of equations. The full resolvent is

$$
\begin{aligned}
G & =\left(E+i 0-H_{0}-\sum_{\gamma} v_{\gamma}-\sum_{\gamma} u_{\gamma}\right)^{-1}, \\
G & =G_{\beta}+G_{\beta} \sum_{\gamma}\left(\bar{\delta}_{\beta \gamma} v_{\gamma}+u_{\gamma}\right) G,
\end{aligned}
$$

where $\bar{\delta}_{\beta \alpha}=1-\delta_{\beta \alpha}, E$ is the available three-particle energy and $H_{0}$ the three-particle kinetic energy operator, both in the center-of-mass (c.m.) system, and the channel resolvents are

$$
\begin{aligned}
& G_{\gamma}=\left(E+i 0-H_{0}-v_{\gamma}\right)^{-1}, \\
& G_{\gamma}=G_{0}+G_{0} T_{\gamma} G_{0} .
\end{aligned}
$$

Here $G_{0}=\left(E+i 0-H_{0}\right)^{-1}$ is the free resolvent and

$$
T_{\gamma}=v_{\gamma}+v_{\gamma} G_{0} T_{\gamma}
$$

the two-particle transition matrix.

The multichannel three-particle transition operators $U_{\beta \alpha}$ are defined by the decomposition of the full resolvent into channel resolvents according to

$$
G=\delta_{\beta \alpha} G_{\alpha}+G_{\beta} U_{\beta \alpha} G_{\alpha} .
$$

Inserting Eq. (5) with $\beta=\gamma$ into Eq. (2b), comparing it back to Eq. (5) and taking into account Eqs. (3a) and (4) 
we obtain integral equation for the multichannel threeparticle transition operators

$$
\begin{aligned}
U_{\beta \alpha}= & \bar{\delta}_{\beta \alpha} G_{0}^{-1}+u_{\alpha}+\sum_{\gamma} \bar{\delta}_{\beta \gamma} T_{\gamma} G_{0} U_{\gamma \alpha} \\
& +\sum_{\gamma} u_{\gamma} G_{0}\left(1+T_{\gamma} G_{0}\right) U_{\gamma \alpha} .
\end{aligned}
$$

The on-shell matrix elements $\left\langle\phi_{\beta}\left|U_{\beta \alpha}\right| \phi_{\alpha}\right\rangle$ are amplitudes (up to a factor) for elastic $(\beta=\alpha)$, rearrangement $(0 \neq \beta \neq \alpha)$ scattering, and breakup $(\beta=0)$. The channel states $\left|\phi_{\alpha}\right\rangle$ are the eigenstates of the corresponding channel Hamiltonian $H_{\alpha}=H_{0}+v_{\alpha}$ with the energy eigenvalue $E$ while $\left|\phi_{0}\right\rangle$ describes the free relative motion of three particles; the dependence on the Jacobi momenta and discrete quantum numbers is suppressed in our notation.

For nucleon-deuteron scattering it is convenient to consider nucleons as identical particles in the isospin formalism where the symmetrized amplitudes are $\sum_{\alpha}\left\langle\phi_{\beta}\left|U_{\beta \alpha}\right| \phi_{\alpha}\right\rangle$. The symmetrized transition operator for elastic scattering is the solution of the symmetrized AGS integral equation

$$
\begin{aligned}
U= & P G_{0}^{-1}+(1+P) u+P T G_{0} U \\
& +(1+P) u G_{0}\left(1+T G_{0}\right) U
\end{aligned}
$$

We omit the spectator index that is not needed anymore. The basis states are antisymmetric in the pair only, the full antisymmetry is ensured by $P=P_{12} P_{23}+P_{13} P_{23}$ where $P_{\alpha \beta}$ is the permutation operator of particles $\alpha$ and $\beta$. The breakup operator is then obtained from the quadrature

$$
U_{0}=(1+P)\left[G_{0}^{-1}+u+T G_{0} U+u G_{0}\left(1+T G_{0}\right) U\right] .
$$

For the practical solution it is convenient to introduce the transition operator

$$
X=G_{0}^{-1}+u+T G_{0} U+u G_{0}\left(1+T G_{0}\right) U
$$

such that

$$
\begin{aligned}
U & =\left[P+u G_{0}(1+P)\right] X, \\
U_{0} & =(1+P) X .
\end{aligned}
$$

Operator $X$ is obtained from the integral equation

$$
X=G_{0}^{-1}+T G_{0} P X+\left(1+T G_{0}\right) u G_{0}(1+P) X .
$$

The practical advantage of this equation is that all terms have the same structure with respect to the relative momentum of the interacting pair in the final state; this is convenient for the interpolation that is needed to calculate $P X$ at each iteration step. The handling of the $3 N$ force is discussed in the Appendix $\mathrm{A}$. Otherwise, the numerical technique for solving Eq. (10) in momentumspace partial-wave representation and calculating onshell elements in Eqs. (9) is taken over from Refs. [16, 17].
There, two equally reliable interpolation methods for the two-nucleon transition matrices were used. However, in the presence of the irreducible $3 N$ force we use the one based on splines that is more convenient than the one based on the Chebyshev expansion.

Finally we note that in Refs. [1, 2] nucleon-deuteron scattering equations of similar form were solved. The relation between our transition operator $X$ and the operator $T$ of Refs. [1, 2], not to be confused with the twonucleon transition matrix $T$ in the equations above, reads $X=G_{0}^{-1}+T$.

\section{INCLUSION OF THE COULOMB FORCE}

In order to include the Coulomb interaction we use the method of screening and renormalization [13, 14] as described in detail in Refs. [11, 18, 19] for pairwise interactions. The screened Coulomb potential $w_{\gamma R}$, that in the configuration space representation has the form $w_{\gamma R}(r)=\left(\alpha_{e} / r\right) \exp \left(-(r / R)^{n}\right), \alpha_{e} \approx 1 / 137$ being the fine structure constant, is added to the hadronic protonproton $(p p)$ potential $v_{\gamma}$. The $p p$ transition matrix (4) is calculated with the full interaction $v_{\gamma}+w_{\gamma R}$ and used to solve the AGS equations yielding multichannel transition operators $U_{\beta \alpha}^{(R)}\left(U^{(R)}\right.$ and $U_{0}^{(R)}$ in the symmetrized version); their dependence on the Coulomb screening radius $R$ is indicated. Following the strategy of Refs. [11, 18, 19] it is straightforward to decompose the amplitudes into long-range and Coulomb-distorted short-range parts also when the $3 N$ force is present. The only difference is that the integral equations for the reduced short-range operators $\tilde{U}_{\beta \alpha}^{(R)}$ of Refs. [11, 18, 19] contain additional three-body force terms, i.e.,

$$
\begin{aligned}
\tilde{U}_{\beta \alpha}^{(R)}= & \bar{\delta}_{\beta \alpha}\left(G_{\alpha R}^{-1}+v_{\alpha}\right)+u_{\alpha}+\delta_{\beta \alpha} \mathcal{W}_{\alpha R} \\
& +\sum_{\gamma}\left(\bar{\delta}_{\beta \gamma} v_{\gamma}+u_{\gamma}+\delta_{\beta \gamma} \mathcal{W}_{\beta R}\right) G_{\gamma R} \tilde{U}_{\gamma \alpha}^{(R)}, \\
\tilde{U}_{0 \alpha}^{(R)}= & G_{\alpha R}^{-1}+v_{\alpha}+u_{\alpha}+\sum_{\gamma}\left(v_{\gamma}+u_{\gamma}\right) G_{\gamma R} \tilde{U}_{\gamma \alpha}^{(R)},
\end{aligned}
$$

where $G_{\alpha R}$ and $\mathcal{W}_{\alpha R}$ are defined in Refs. [11, 18, 19]. Nevertheless, the relation of $\tilde{U}_{\beta \alpha}^{(R)}$ to the full AGS operators $U_{\beta \alpha}^{(R)}$ as well as the screened Coulomb contributions diverging in the $R \rightarrow \infty$ limit remain the same. Thus, the renormalization prescription can be taken over from Refs. [11, 18, 19] where the amplitudes for $p d$ elastic scattering and breakup referring to unscreened Coulomb are calculated as

$$
\begin{aligned}
\left\langle\phi^{\prime}\left|U^{(C)}\right| \phi\right\rangle & =\left\langle\phi^{\prime}\left|T_{C}^{c . m} \cdot\right| \phi\right\rangle \\
& +\lim _{R \rightarrow \infty}\left\{\mathcal{Z}_{R}^{-1}\left(q_{i}\right)\left\langle\phi^{\prime}\left|\left[U^{(R)}-T_{R}^{\text {c.m. }} \cdot\right]\right| \phi\right\rangle\right\}, \\
\left\langle\phi_{0}\left|U_{0}^{(C)}\right| \phi\right\rangle & =\lim _{R \rightarrow \infty}\left\{z_{R}^{-\frac{1}{2}}\left(p_{f}\right)\left\langle\phi_{0}\left|U_{0}^{(R)}\right| \phi\right\rangle \mathcal{Z}_{R}^{-\frac{1}{2}}\left(q_{i}\right)\right\} .
\end{aligned}
$$


The long-range part of the screened elastic scattering amplitude is given by the two-body on-shell transition matrix $\left\langle\phi^{\prime}\left|T_{R}^{\text {c.m. }}\right| \phi\right\rangle$ derived from the screened Coulomb potential between proton and the c.m. of the deuteron. Renormalized by $\mathcal{Z}_{R}^{-1}\left(q_{i}\right)$ in the $R \rightarrow \infty$ limit it converges (in general, as a distribution) to the well-known pure Coulomb amplitude $\left\langle\phi^{\prime}\left|T_{C}^{\mathrm{c} . \mathrm{m}}\right| \phi\right\rangle$. The Coulombdistorted short-range parts $\left\langle\phi^{\prime}\left|\left[U^{(R)}-T_{R}^{\text {c.m. }}\right]\right| \phi\right\rangle$ and $\left\langle\phi_{0}\left|U_{0}^{(R)}\right| \phi\right\rangle$ are calculated numerically at finite $R$ since after the renormalization they rapidly converge with $R$ due to their short-range nature; we only have to make sure that $R$ is large enough for the desired accuracy. In Eqs. (12) $q_{i}$ is the magnitude of the initial relative $p d$ momentum and $p_{f}$ is the magnitude of the final relative $p p$ momentum. The renormalization factors $\mathcal{Z}_{R}\left(q_{i}\right)$ and $z_{R}\left(p_{f}\right)$ are diverging phase factors defined in Ref. [13] for a general screening and given in Refs. [11, 18, 19] for the form of screening used there as well as in this work. We get well-converged results for the observables when the Coulomb-distorted short-range part of the amplitudes is calculated using the screening function with $n=4$ and $R=10 \mathrm{fm}(30 \mathrm{fm})$ for $p d$ elastic scattering (breakup). With those values of $R$ the partial-wave expansion converges slower than in the $n d$ case but the AGS equations still can be solved in the partial-wave basis. In our first calculations with Coulomb [11, 18] high two-baryon partial waves needed for the convergence were included using the perturbative approach of Ref. 20] that was confirmed to be highly reliable in later calculations where we found an efficient method 21] to include the high partial waves exactly. This latter method [21] is used in the present work where we obtain fully converged results by taking into account the hadronic interaction in two-nucleon partial waves with total pair angular momentum $I \leq 5$ and the screened Coulomb interaction in two-proton partial waves with pair orbital angular momentum $L \leq 14$. The partial waves with total $3 N$ angular momentum $\mathcal{J} \leq \frac{59}{2}$ are considered but it is fully sufficient to include the $3 N$ force only in those with $\mathcal{J} \leq \frac{19}{2}$. Of course, both total $3 N$ isospin $\mathcal{T}=\frac{1}{2}$ and $\frac{3}{2}$ states are included.

Thus, the presence of the $3 N$ force does not change the rate of the $R$-convergence for the observables whose detailed study was given in Refs. [11, 18] and will not be repeated here. However, a recent work [22] on an alternative Coulomb treatment in $p d$ scattering proposed a renormalization prescription that is different from ours given in Eqs. (12). According to Ref. 22], the $p d$ elastic scattering amplitude calculated with screened Coulomb does not need renormalization at all, i.e., the limit $\lim _{R \rightarrow \infty}\left\langle\phi^{\prime}\left|U^{(R)}\right| \phi\right\rangle$ should exist. The renormalization for the breakup amplitude is needed but it is different from ours given in Eq. (12b). However, numerical results of Ref. [22] involve approximations that are not well under control: the screened Coulomb transition matrix is approximated by the screened Coulomb potential and, furthermore, particular contributions to the $p d$ scattering amplitudes that include first order terms in the screened Coulomb transition matrix are neglected.
Therefore we feel the need to clarify the issue of renormalization. We study the dependence on the screening radius $R$ for selected components of the nonrenormalized and renormalized $p d$ elastic scattering amplitudes, $\left\langle\phi^{\prime}\left|U^{(R)}\right| \phi\right\rangle$ and $\left\langle\phi^{\prime}\left|U^{(C)}\right| \phi\right\rangle$, calculated at proton lab energy $E_{p}=9 \mathrm{MeV}$; the AV18 $N N$ potential [8] and the Urbana IX $3 N$ force [9] are taken as the hadronic interaction. In Fig. 1 we show spin-nondiagonal amplitudes that have only the Coulomb-distorted short-range part. The nonrenormalized amplitude $\left\langle\phi^{\prime}\left|U^{(R)}\right| \phi\right\rangle$ shows a clear $R$-dependence; in fact, its absolute value becomes $R$-independent but the phase is proportional to $\ln R$. In contrast, the renormalized amplitude $\left\langle\phi^{\prime}\left|U^{(C)}\right| \phi\right\rangle$, within the accuracy of the plot, becomes independent of $R$ for $R \geq 10 \mathrm{fm}$. The renormalized spin-diagonal amplitude that has also a long-range part becomes $R$-independent as well, as Fig. 2 demonstrates. Thus, fully converged numerical results without uncontrolled approximations clearly support the standard screening and renormalization theory as given in Eqs. (12) and not the one of Ref. [22].

\section{RESULTS}

Numerical results of this paper are derived from the AV18 $N N$ potential [8] together with the Urbana IX $3 N$ force [9]; this combination, AV18 + UIX, is one of the most widely used $N N+3 N$ force models. Hadronic charge dependence is fully included, i.e., $p p$ and $n p$ potentials are used within the isospin formalism. Point Coulomb interaction is added for $p p$ but the additional electromagnetic terms of AV18 are not included. In order to isolate Coulomb and $3 N$ force effects, calculations without Coulomb or without the $3 N$ force are performed as well. Obviously, we have many more predictions than it is possible and wise to show. Therefore we make a judicious selection and present the most interesting cases. The readers, dissatisfied with our choice, are welcome to obtain the results for their favorite data from us.

In Fig. 3 we show results for the differential cross section and analyzing powers of $p d$ elastic scattering at $9 \mathrm{MeV}$ proton lab energy (18 MeV deuteron lab energy). The Coulomb effect is important in the whole kinematical regime. There are well-known discrepancies [6] around the maximum of the vector analyzing powers $A_{y}(N)$ and $i T_{11}$ and the first minimum of $T_{21}$. The $3 N$ force effect is rather small; it is mostly a scaling effect due to the change in the binding energies of ${ }^{3} \mathrm{H}$ and ${ }^{3} \mathrm{He}$ that are calculated in the Appendix B In addition, observables shown in Fig. 3 serve as a benchmark since they already have been calculated in Ref. [6] using AV18 and AV18 + UIX force models together with the Coulomb force. The $3 N$ Schrödinger equation was solved in configuration space using the Kohn variational principle and explicitly imposing the proper Coulomb boundary conditions. The $p d$ elastic scattering results obtained with the two methods for including the $p p$ Coulomb interaction, i.e., our 

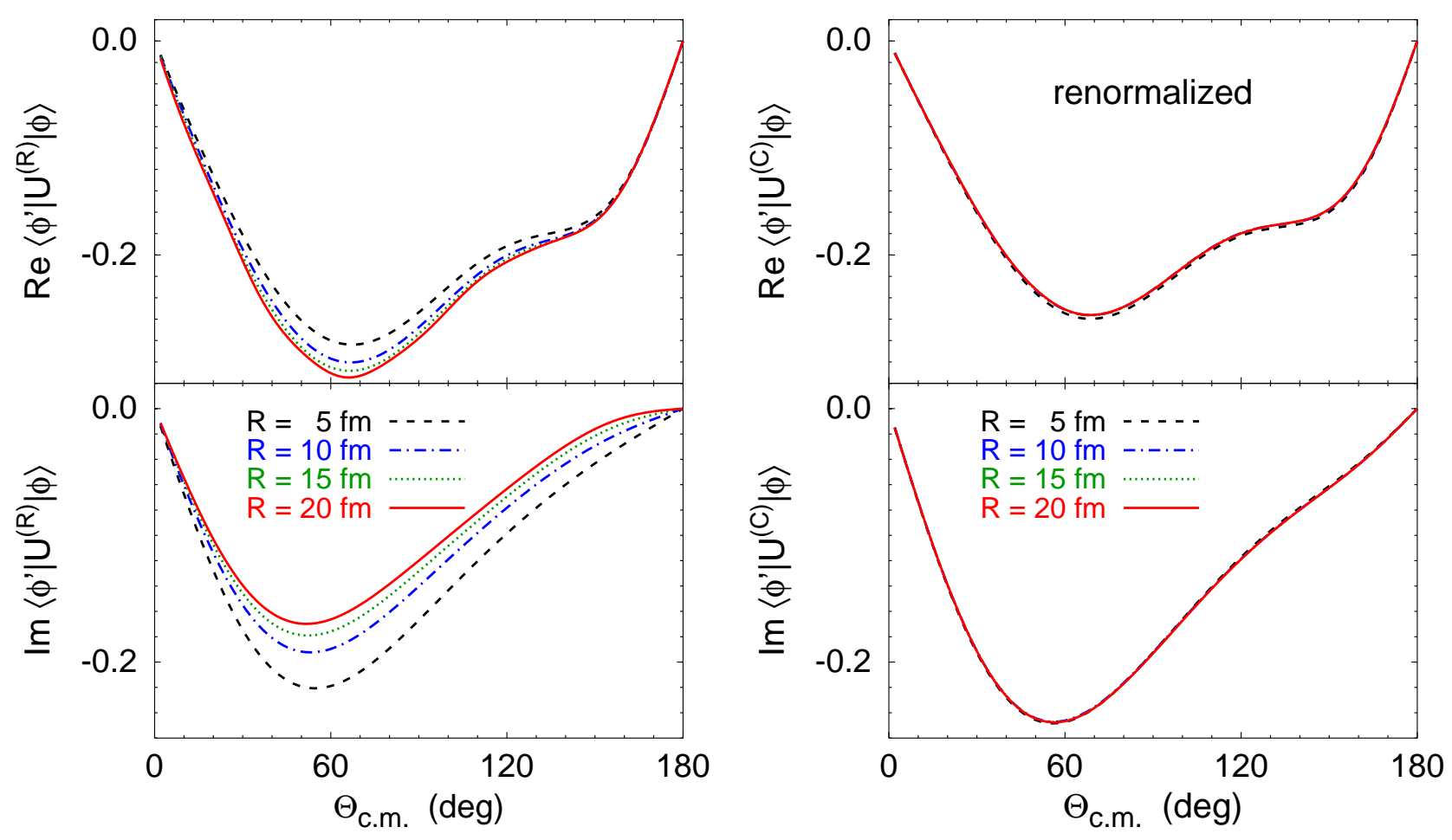

FIG. 1. (Color online) Real and imaginary parts of nonrenormalized (left side) and renormalized (right side) pd elastic scattering amplitudes (in arbitrary units) at $E_{p}=9 \mathrm{MeV}$ shown as functions of the c.m. scattering angle. The initial and final particle spin projection quantum numbers are $m_{p}=\frac{1}{2}, m_{p}^{\prime}=-\frac{1}{2}$, and $m_{d}=m_{d}^{\prime}=1$. Results for the AV18 + UIX force model obtained with the screening radius $R=5 \mathrm{fm}$ (dashed curves), $10 \mathrm{fm}$ (dash-dotted curves), $15 \mathrm{fm}$ (dotted curves), and $20 \mathrm{fm}$ (solid curves) are compared.

momentum-space screening and renormalization method and the one of Ref. [6], were compared in Ref. [24] taking the AV18 NN potential as the hadronic interaction alone; a good agreement over a wide range of energies for all studied observables was found. Comparing Fig. 3 to the $9 \mathrm{MeV}$ results of Ref. [6] it is easy to see that the predicted $3 N$ force effect is the same in both cases. Thus, the agreement between the momentum-space and configuration-space results including both the $3 N$ force and Coulomb and is as good as without the $3 N$ force.

In Fig. 4 we show the differential cross section and analyzing powers for $p d$ elastic scattering at $135 \mathrm{MeV}$ proton lab energy (270 MeV deuteron lab energy) where the configuration-space calculations are not available so far. As found in Ref. [11] and confirmed in the present work, the Coulomb effect at this relatively high energy is large only at forward angles. We therefore do not show it separately. Instead, since the $3 N$ force effect is quite significant, it is interesting to compare two different $3 N$ force models: (a) Urbana IX, based on the Fujita-Miyazawa force [27], i.e., the two-pion $(2 \pi)$ exchange with an intermediate $\Delta$-isobar excitation, and supplemented by a purely phenomenological repulsive short-range part; (b) an effective $3 N$ force due to explicit $\Delta$-isobar excitation that uses no static approximation for the propagation of the $\Delta$, includes beside the pion also the exchange of heavier mesons $\rho, \omega$, and $\sigma$, and has higher order contributions, e.g., three-meson ring diagrams; all contributions are consistent with each other since they are built from the same two-baryon coupled-channel potential CD Bonn $+\Delta[3]$ that is as realistic as its purely nucleonic reference potential CD Bonn [28]. The $\Delta$-isobar effect is isolated as the difference between predictions of the CD Bonn $+\Delta$ and CD Bonn potentials. In most cases both Urbana IX and $\Delta$-isobar effects are qualitatively similar although the former is usually larger, especially around the minimum of the deuteron vector analyzing power $A_{y}(d)$ where the predictions of AV18 + UIX are considerably closer to the experimental data. The reason why the $\Delta$-isobar effect is smaller, at least partially, is the $N N$ dispersion that is generated by the explicit $\Delta$-isobar excitation and often competes with the effective $3 N$ force [3]. The two $3 N$ force models show qualitatively different behavior around the first minimum of the deuteron tensor analyzing power $A_{x x}$ where the CD Bonn $+\Delta$ accounts for the data better. Finally we note that our AV18 + UIX results without Coulomb (not shown here) are consistent with the corresponding results of Ref. [1].

In Fig. [5] we show the fivefold differential cross section for $p d$ breakup at $13 \mathrm{MeV}$ proton lab energy in few special kinematical configurations that are characterized in a standard way by the final-state polar angles 


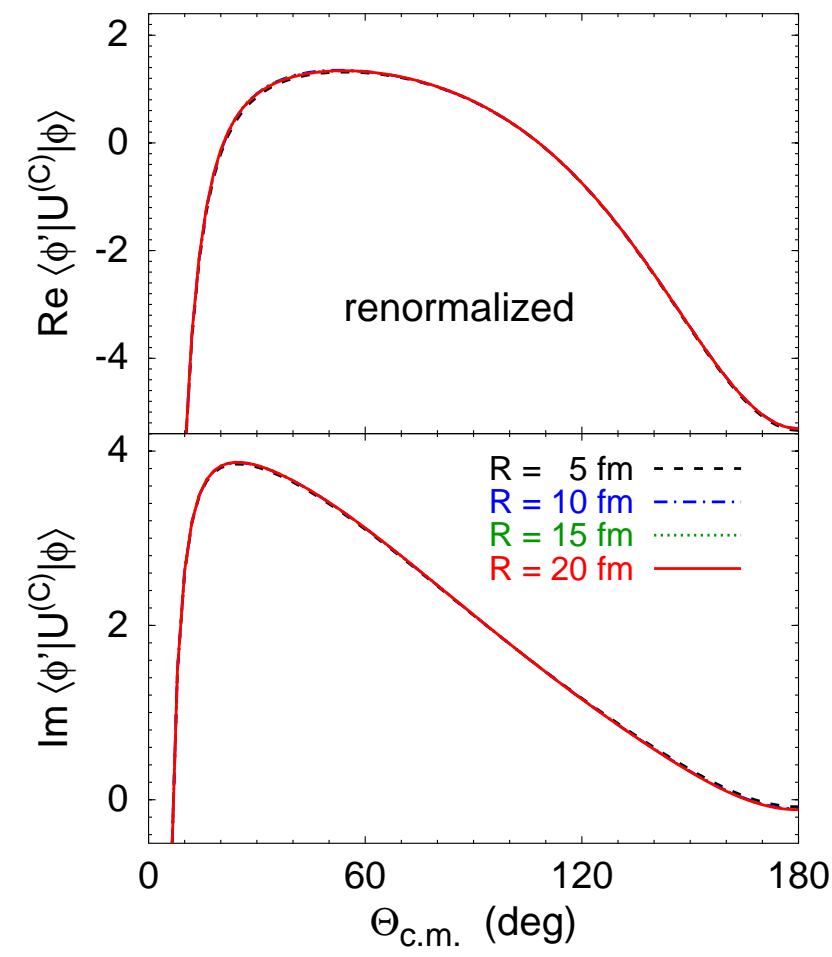

FIG. 2. (Color online) Real and imaginary parts of the renormalized $p d$ elastic scattering amplitude. The initial and final particle spin projection quantum numbers are $m_{p}=m_{p}^{\prime}=\frac{1}{2}$, and $m_{d}=m_{d}^{\prime}=1$. Curves as in Fig. 1]

of the two detected protons and by the azimuthal angle between them, $\left(\theta_{1}, \theta_{2}, \varphi_{12}=\varphi_{2}-\varphi_{1}\right)$. Though the inclusion of Coulomb slightly improves the agreement with data in the space star configuration, the Coulomb effect is far too small to reproduce the difference between the experimental $p d$ and $n d$ data and to resolve the socalled space star anomaly. Slightly larger and beneficial Coulomb effects are seen in quasifree scattering (QFS) and collinear configurations; the differential cross section is decreased around QFS peak and increased around the collinear point (minimum) and $n p$ final-state interaction (FSI) peaks. The remaining discrepancies around the $n p$-FSI peaks, at least to some extent, may be due to the finite geometry, not taken into account in our calculations owing to the lack of information on experimental details. The $3 N$ force effect is very small except for the QFS configuration. All these findings are consistent with our previous results [18] derived from the CD Bonn $+\Delta$ and CD Bonn potentials.

Finally, we consider $p d$ breakup at $130 \mathrm{MeV}$ deuteron lab energy that was measured recently in a variety of kinematical configurations [32, 33]. In some of them we found sizable Coulomb effects for the differential cross section [18, 33] but very small $\Delta$-isobar effects. In contrast, the present calculations as well as those without Coulomb given in Ref. 32] reveal visible effects of the Urbana IX $3 N$ force. Therefore a more extensive study of the interplay between the Coulomb and the $3 N$ force is needed. In Fig. 6 we show the fivefold differential cross section for few kinematical configurations with small relative azimuthal angle $\varphi_{12}$. The central point of the $\left(13^{\circ}, 13^{\circ}, 20^{\circ}\right)$ configuration corresponds to very low relative $p p$ energy in the final state, $E_{p p}<0.2 \mathrm{MeV}$, where the differential cross section and also the $3 N$ force effect are strongly reduced by the Coulomb as a result of the $p p$ repulsion; the found Coulomb effect is well supported by the experimental data. The relative $p p$ energy in the final state increases with the relative polar and azimuthal angles of the protons and therefore the Coulomb effect decreases in the remaining configurations of Fig. 6] but the $3 N$ force effect becomes more visible. The relative $p p$ energy gets larger at $\varphi_{12}=160^{\circ}$ in Fig. 7 leading to an increase of the differential cross section due to the Coulomb, especially in the configurations with smaller $\theta_{i}$. Since the total breakup cross section at this energy is almost unchanged by Coulomb as demonstrated in Ref. [18] and confirmed in the present work, one may expect in particular configurations an increase of the differential cross section due to Coulomb to compensate for the strong decrease in the regions with low $E_{p p}$. The inclusion of the Urbana IX $3 N$ force, as in Fig. 6, increases the differential cross section, especially in the configurations where both $\theta_{i}$ are large. Thus, at smaller $\theta_{i}$ Coulomb is dominating while at larger $\theta_{i}$ the Urbana IX $3 N$ force becomes more significant. In some kinematical regimes, e.g., around the central peaks of the configurations $20^{\circ} \leq \theta_{1} \leq 30^{\circ}, \theta_{2}=20^{\circ}, \varphi_{12}=160^{\circ}$, both are equally important leading to quite a satisfactory description of the data in all studied configurations.

Deuteron analyzing powers were measured in the same experiments [32, 33], however, the data analysis is not yet completed. We therefore present only few examples in Fig. 8 demonstrating that for the spin observables like $A_{x x}$ Coulomb and $3 N$ force effects may take place in completely different regions of the phase space compared to the differential cross section. E.g., $A_{x x}$ remains unaffected by the Coulomb in the $\left(13^{\circ}, 13^{\circ}, 20^{\circ}\right)$ configuration with very low $E_{p p}$ where a strong decrease of the cross section was found in Fig. 6, but shows a moderate Coulomb effect in the $\left(25^{\circ}, 20^{\circ}, 40^{\circ}\right)$ configuration where it was almost negligible in the case of cross section. In contrast, the $3 N$ force effect in the latter configuration is only significant for the differential cross section but not for $A_{x x}$. The last configuration in Fig. 8 shows moderate effects of both Coulomb and $3 N$ force competing with each other unlike in the case of the differential cross section in Fig. 7. A detailed study of deuteron analyzing powers in more kinematical configurations is postponed till the finalization of the experimental data that is expected soon [34]. 

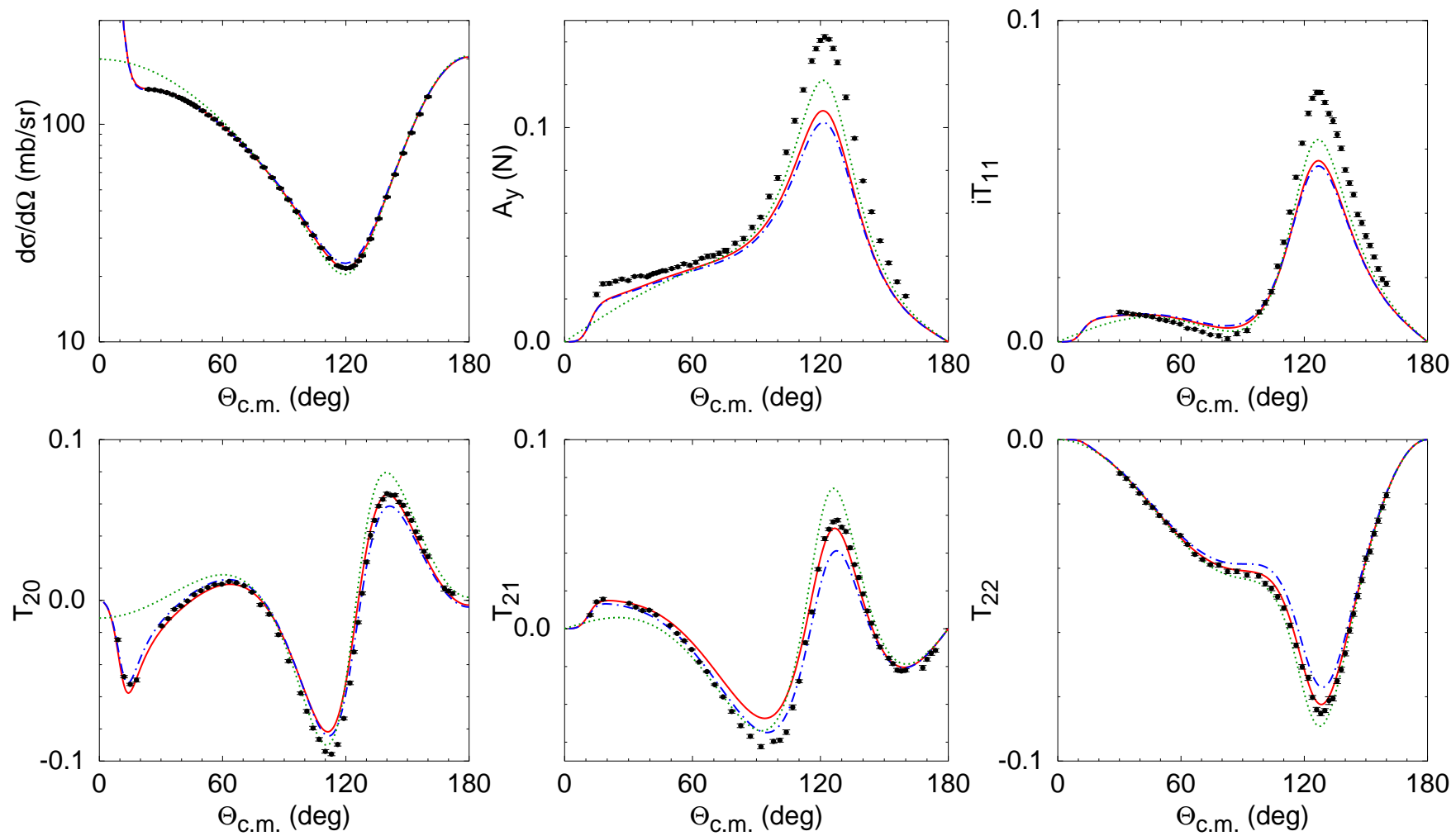

FIG. 3. (Color online) Differential cross section and analyzing powers for $p d$ elastic scattering at 9 MeV proton lab energy as functions of the c.m. scattering angle. Results for AV18 (dashed-dotted curves) and AV18 + UIX (solid curves) force models, both including Coulomb, and for AV18 + UIX without Coulomb (dotted curves) are compared with the experimental data from Ref. [23].

\section{SUMMARY}

In this paper we derive AGS integral equations for proton-deuteron scattering including an irreducible three-nucleon force and solve them in the momentumspace partial wave representation. The Coulomb interaction between the protons is included using the screening and renormalization method whose validity is confirmed by the numerical results for scattering amplitudes; they are well converged with respect to the screening and with respect to the quantum number cutoffs. AV18 NN potential with the Urbana IX $3 N$ force are used as the hadronic interaction model to calculate the observables of $p d$ elastic scattering and breakup. In the low-energy $p d$ elastic scattering, where configuration-space calculations [6] with the same dynamic input are available, a good agreement between our results and those of Ref. [6] is found. For $p d$ elastic scattering at higher energies and for $p d$ breakup we provide first results for the AV18 + UIX force model with Coulomb. In higher-energy $p d$ elastic scattering where the Coulomb effect is confined to small scattering angles but the $3 N$ force effect is significant, we compared two models, Urbana IX and the effective $3 N$ force due to explicit $\Delta$-isobar excitation, and with few exceptions found a qualitative agreement between them.
In breakup the Coulomb effects are fully consistent with those found in our earlier calculations [11, 18] with different hadronic interactions. The inclusion of Coulomb is unable to resolve the space star anomaly at low-energies, but clearly improves the description of the experimental data at $130 \mathrm{MeV}$ deuteron lab energy where, depending on the kinematical configuration, it may significantly decrease or increase the differential cross section. A moderate Urbana IX $3 N$ force effect is seen as well that increases the cross section which is only slightly underpredicted by the theory. A complicated interplay of Coulomb and $3 N$ force effects takes place in the spin observables; they will be studied extensively in the future using other $3 \mathrm{~N}$ force models as well.

\section{ACKNOWLEDGMENTS}

The author thanks R. Lazauskas for valuable discussions on the inclusion of the $3 N$ force, and A. C. Fonseca for the comments on the manuscript. 

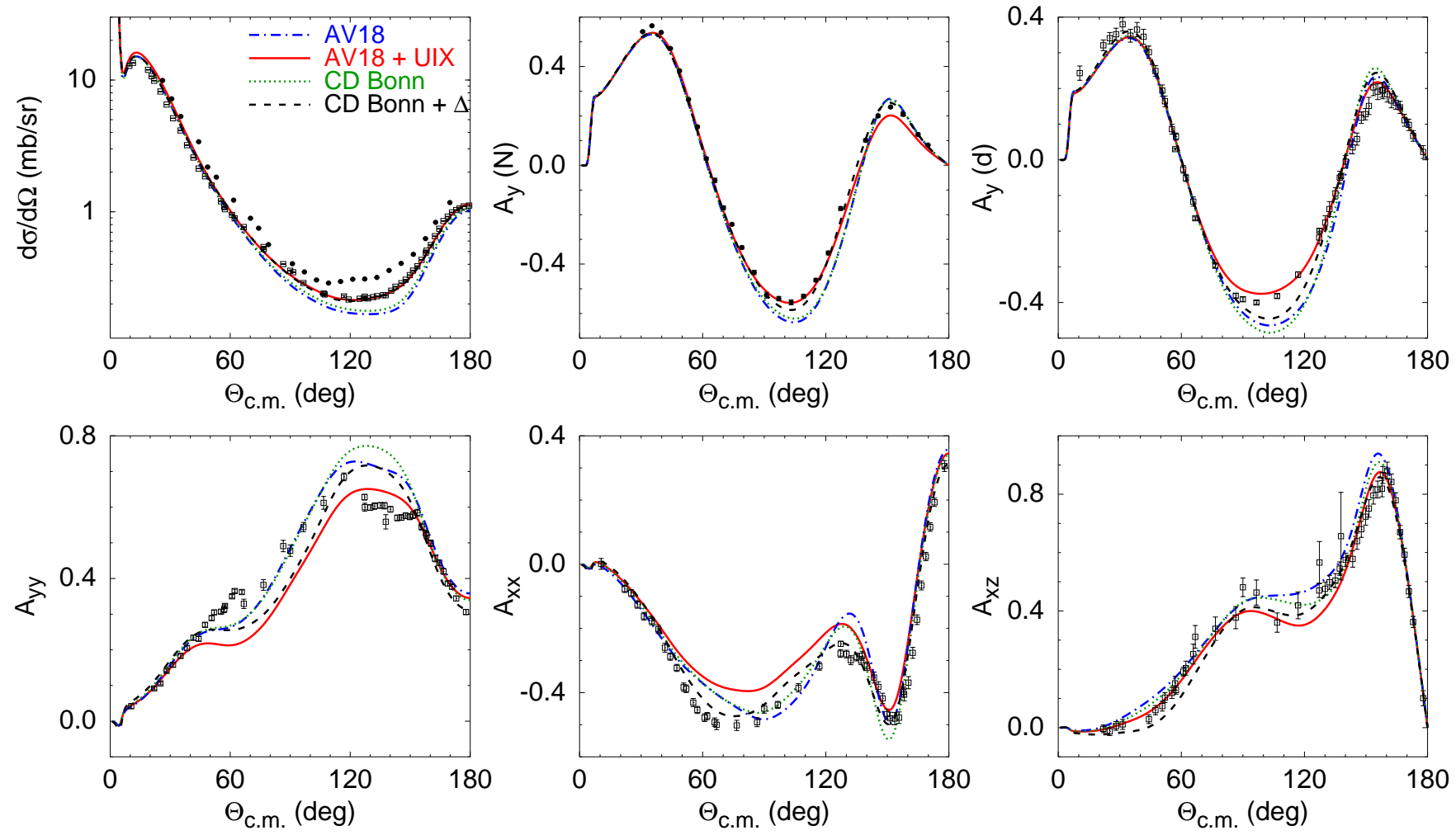

FIG. 4. (Color online) Differential cross section and analyzing powers for $p d$ elastic scattering at $135 \mathrm{MeV}$ proton lab energy. Results for AV18 (dashed-dotted curves), AV18 + UIX (solid curves), CD Bonn (dotted curves), and CD Bonn $+\Delta($ dashed curves) force models, all including Coulomb, are compared with the experimental data from Ref. [25] (full circles) and Ref. [26] (open squares).
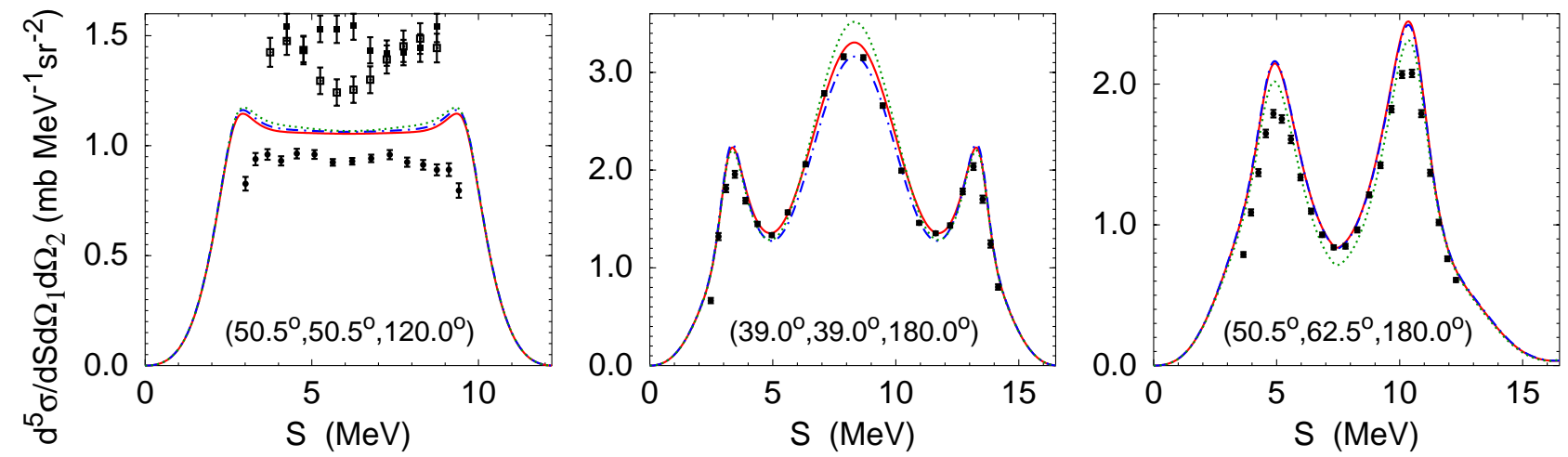

FIG. 5. (Color online) Differential cross section for $p d$ breakup at $13 \mathrm{MeV}$ proton lab energy in space star (left), quasifree scattering (middle), and collinear (right) configurations as function of the arclength $S$ along the kinematical curve. Curves as in Fig. 33 and the experimental data from Ref. [29] (full circles). For the space star configuration also the nd data from Refs. [30, 31] (open and full squares) are shown.

\section{Appendix A: Urbana IX $3 N$ force}

The Urbana IX $3 N$ force has $2 \pi$-exchange and phenomenological repulsive short-range terms,

$$
\begin{aligned}
V_{(3)}= & \sum_{\alpha \beta \gamma \text { cyclic }}\left(A_{2 \pi}\left\{X_{\alpha \beta}^{\pi}, X_{\beta \gamma}^{\pi}\right\}\left\{\tau_{\alpha} \cdot \tau_{\beta}, \tau_{\beta} \cdot \tau_{\gamma}\right\}\right. \\
& \left.+C_{2 \pi}\left[X_{\alpha \beta}^{\pi}, X_{\beta \gamma}^{\pi}\right]\left[\tau_{\alpha} \cdot \tau_{\beta}, \tau_{\beta} \cdot \tau_{\gamma}\right]+U_{0} T_{\alpha \beta}^{2} T_{\beta \gamma}^{2}\right),
\end{aligned}
$$

where curly and square brackets denote anticommutator and commutator, respectively. The strength constants are $A_{2 \pi}=-0.0293 \mathrm{MeV}, C_{2 \pi}=\frac{1}{4} A_{2 \pi}$, and $U_{0}=0.0048$ $\mathrm{MeV}$; the latter should be not confused with the symmetrized breakup operator $U_{0}$ defined in Sec. III $\tau_{\alpha}$ is the isospin vector operator of the nucleon $\alpha$, and $X_{\alpha \beta}^{\pi}$ 

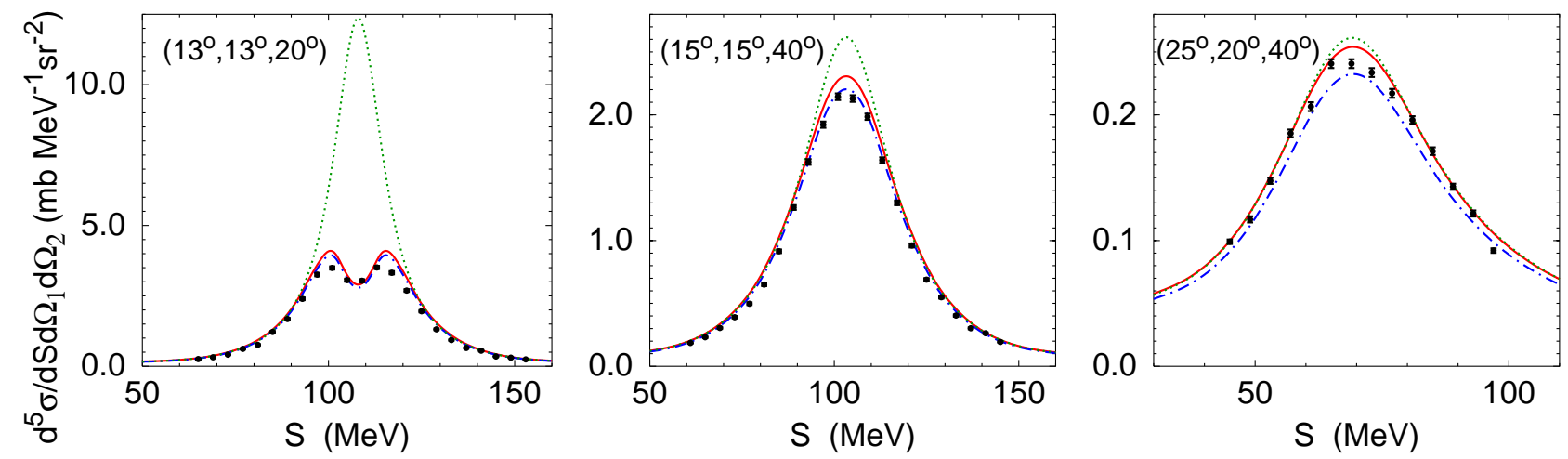

FIG. 6. (Color online) Differential cross section for $p d$ breakup at $130 \mathrm{MeV}$ deuteron lab energy in selected kinematical configurations with small relative azimuthal angle. Curves as in Fig. 3] and the experimental data from Refs. 32, 33].
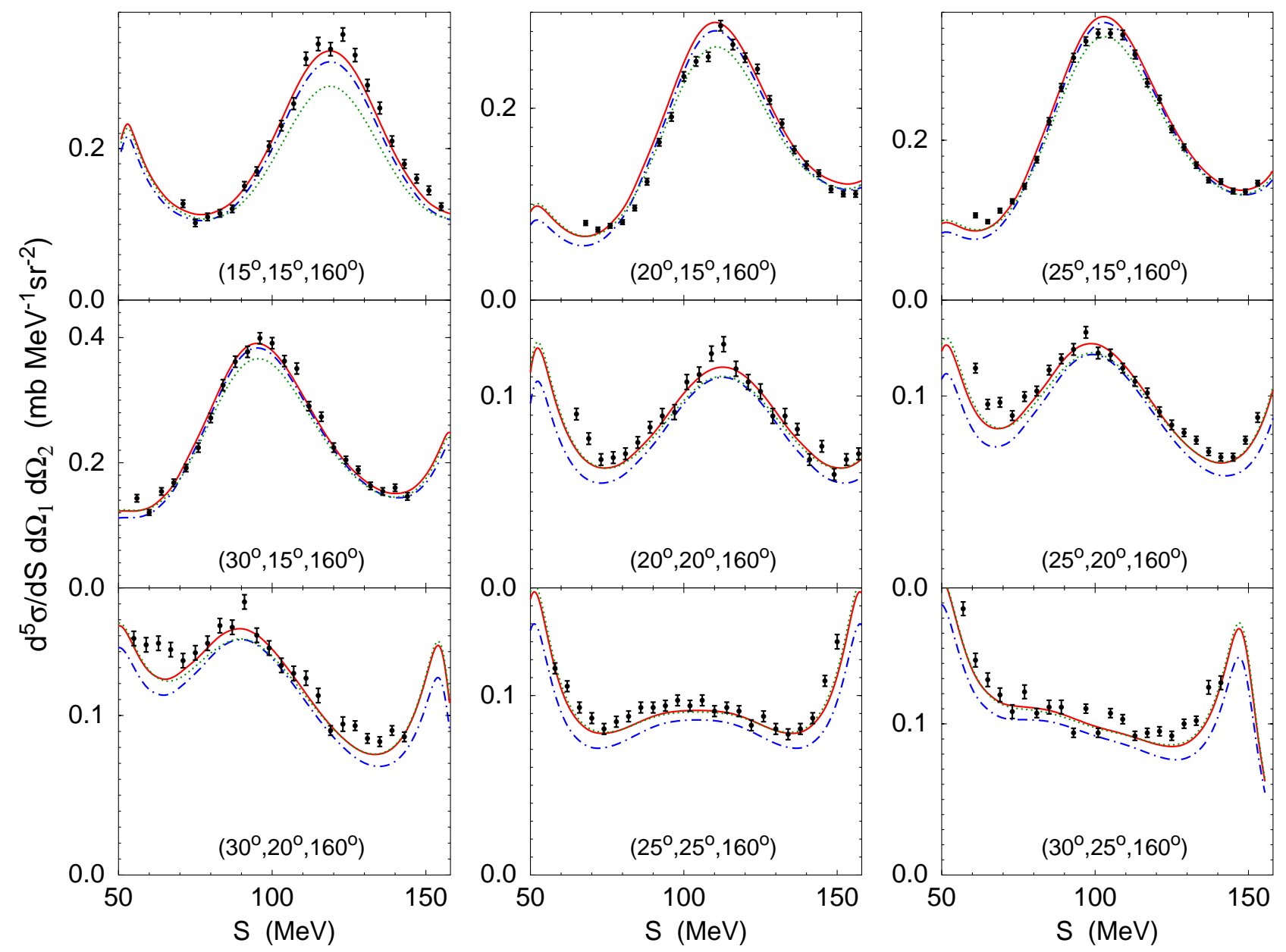

FIG. 7. (Color online) Differential cross section for $p d$ breakup at $130 \mathrm{MeV}$ deuteron lab energy in selected kinematical configurations with large relative azimuthal angle. Curves as in Fig. 3 and the experimental data from Ref. 32].

and $T_{\alpha \beta}$ are local potential-like two-nucleon operators depending on the relative coordinate of the nucleons $\alpha$ and $\beta$ and, in the case of $X_{\alpha \beta}^{\pi}$, also on their spins; the explicit expressions can be found in Ref. [9]. The transformation of $X_{\alpha \beta}^{\pi}$ and $T_{\alpha \beta}^{2}$ to the momentum space using spherical Bessel functions is straightforward. For decomposing 

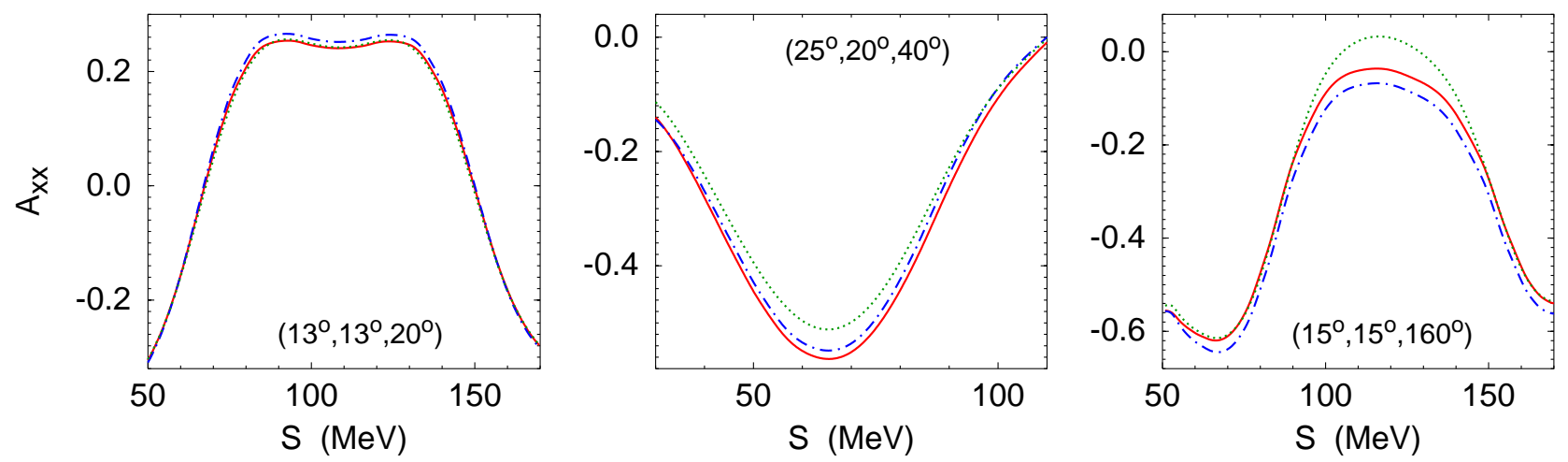

FIG. 8. (Color online) Deuteron analyzing power $A_{x x}$ for $p d$ breakup at $130 \mathrm{MeV}$ deuteron lab energy in selected kinematical configurations. Curves as in Fig. 3 ,

(A1) into three symmetric parts we follow Ref. [35], i.e.,

$$
\begin{aligned}
u_{\gamma}= & A_{2 \pi} X_{\alpha \beta}^{\pi}\left\{X_{\beta \gamma}^{\pi}\left[2 \tau_{\alpha} \cdot \tau_{\gamma}-\frac{i}{2} \tau_{\alpha} \cdot\left(\tau_{\beta} \times \tau_{\gamma}\right)\right]\right. \\
& \left.+X_{\gamma \alpha}^{\pi}\left[2 \tau_{\gamma} \cdot \tau_{\beta}+\frac{i}{2} \tau_{\alpha} \cdot\left(\tau_{\beta} \times \tau_{\gamma}\right)\right]\right\} \\
& +\frac{1}{2} U_{0} T_{\alpha \beta}^{2}\left(T_{\beta \gamma}^{2}+T_{\gamma \alpha}^{2}\right)
\end{aligned}
$$

$\alpha \beta \gamma$ being cyclic. In the $3 N$ scattering and bound state equations, (10) and (B3), the $3 N$ force component (A2) always acts on a state $|y\rangle$ that is fully antisymmetric due to $(1+P)$. Furthermore, taking into account that $X_{\alpha \beta}^{\pi}, T_{\alpha \beta}$, and $u_{\gamma}$ are symmetric under exchange of the nucleons $\alpha$ and $\beta$, one can prove that certain terms in Eq. (A2) yield equal contributions [35] and calculate the desired matrix elements of the $3 N$ force component as

$$
\begin{aligned}
\gamma\left\langle\nu\left|u_{\gamma}\right| y\right\rangle= & 2 A_{2 \pi} \sum_{\nu_{1} \nu_{2} \nu_{3}} \gamma\left\langle\nu\left|X_{\alpha \beta}^{\pi}\right| \nu_{1}\right\rangle_{\gamma} \\
& \times{ }_{\gamma}\left\langle\nu_{1}\left|\left[2 \tau_{\alpha} \cdot \tau_{\gamma}-\frac{i}{2} \tau_{\alpha} \cdot\left(\tau_{\beta} \times \tau_{\gamma}\right)\right]\right| \nu_{2}\right\rangle_{\alpha} \\
& \times{ }_{\alpha}\left\langle\nu_{2}\left|X_{\beta \gamma}^{\pi}\right| \nu_{3}\right\rangle_{\alpha \alpha}\left\langle\nu_{3} \mid y\right\rangle \\
& +U_{0} \sum_{\nu_{1} \nu_{2} \nu_{3}} \gamma\left\langle\nu\left|T_{\alpha \beta}^{2}\right| \nu_{1}\right\rangle_{\gamma \gamma}\left\langle\nu_{1} \mid \nu_{2}\right\rangle_{\alpha} \\
& \times{ }_{\alpha}\left\langle\nu_{2}\left|T_{\beta \gamma}^{2}\right| \nu_{3}\right\rangle_{\alpha \alpha}\left\langle\nu_{3} \mid y\right\rangle,
\end{aligned}
$$

where each of the intermediate states $\left|\nu_{i}\right\rangle_{\alpha}$, with $\nu_{i}$ abbreviating all continuous and discrete quantum numbers and $\alpha$ denoting the spectator, is antisymmetric under the exchange of the nucleons $\beta$ and $\gamma$. The transformation from the basis with the spectator $\alpha$ to the basis with the spectator $\gamma$ is needed in order to evaluate all the matrix elements of $X_{\alpha \beta}^{\pi}$ and $T_{\alpha \beta}^{2}$ in their proper bases. Since $|y\rangle$ is fully antisymmetric, ${ }_{\alpha}\left\langle\nu_{3} \mid y\right\rangle=$ ${ }_{\gamma}\left\langle\nu_{3} \mid y\right\rangle$. Furthermore, ${ }_{\gamma}\left\langle\nu_{1} \mid \nu_{2}\right\rangle_{\alpha}=\frac{1}{2} \gamma\left\langle\nu_{1}|P| \nu_{2}\right\rangle_{\gamma}$, and ${ }_{\gamma}\left\langle\nu_{1}\left|\left[2 \tau_{\alpha} \cdot \tau_{\gamma}-\frac{i}{2} \tau_{\alpha} \cdot\left(\tau_{\beta} \times \tau_{\gamma}\right)\right]\right| \nu_{2}\right\rangle_{\alpha}$ differs from ${ }_{\gamma}\left\langle\nu_{1} \mid \nu_{2}\right\rangle_{\alpha}$ only by the isospin factor that can be found in Ref. [35]. Thus, the calculation of the $2 \pi$-exchange and short-range terms needs only one basis transformation for each of them, and all intermediate states are antisymmetric with respect to the pair. This constitutes the advantage of the above method [35] for handling the Urbana IX $3 N$ force over the one used in Refs. [1] that needed two basis transformations for each term involving, in addition, intermediate nonphysical symmetric states. Finally we note that for handling other types of $3 N$ forces to be used in the future calculations the technique proposed in Ref. [36] seems to be very promising.

\section{Appendix B: Three-nucleon bound state}

Starting from the Schrödinger equation in the integral form,

$$
|\Psi\rangle=G_{0} \sum_{\alpha}\left(v_{\alpha}+u_{\alpha}\right)|\Psi\rangle
$$

decomposing the $3 N$ bound-state wave function $|\Psi\rangle=$ $\sum_{\alpha}\left|\psi_{\alpha}\right\rangle$ into its Faddeev components $\left|\psi_{\alpha}\right\rangle$ with $\alpha=$ $1,2,3$, and using the definition of the two-particle transition matrix (4) it is straightforward to derive the Faddeev equation

$$
\left|\psi_{\alpha}\right\rangle=G_{0} T_{\alpha} \sum_{\beta} \bar{\delta}_{\beta \alpha}\left|\psi_{\beta}\right\rangle+\left(1+G_{0} T_{\alpha}\right) G_{0} u_{\alpha} \sum_{\beta}\left|\psi_{\beta}\right\rangle
$$

The symmetrized version of it reads

$$
|\psi\rangle=G_{0} T P|\psi\rangle+\left(1+G_{0} T\right) G_{0} u(1+P)|\psi\rangle
$$

where the bound-state wave function is obtained as $|\Psi\rangle=$ $(1+P)|\psi\rangle$. In practical calculations we solve the Faddeev equation for $|x\rangle=G_{0}^{-1}|\psi\rangle$ that has an advantage of having exactly the same form of kernel as the scattering equation (10). In contrast to scattering calculations, we include hadronic interaction in two-nucleon partial waves with total pair angular momentum $I \leq 6$ and take into 
account all electromagnetic terms of the AV18 NN potential. Our results for binding energies and wave function probabilities of ${ }^{3} \mathrm{H}$ and ${ }^{3} \mathrm{He}$ nuclei with and without Urbana IX $3 N$ force are collected in Table If they are in good agreement with the results of other groups [37, 38].

\begin{tabular}{lcccccc}
\hline \hline & $\left|E_{B}\right|$ & $\left\langle H_{0}\right\rangle$ & $P_{S^{\prime}}$ & $P_{P}$ & $P_{D}$ & $P_{\mathcal{T}=3 / 2}$ \\
\hline AV18 & 7.621 & 46.72 & 1.292 & 0.066 & 8.509 & 0.0025 \\
AV18 [37](r) & 7.624 & 46.73 & 1.293 & 0.066 & 8.510 & 0.0025 \\
AV18 [37](p) & 7.621 & 46.73 & 1.291 & 0.066 & 8.510 & 0.0025 \\
AV18+UIX & 8.478 & 51.28 & 1.055 & 0.135 & 9.302 & 0.0025 \\
AV18+UIX [37](r) & 8.479 & 51.28 & 1.054 & 0.135 & 9.301 & 0.0025 \\
AV18+UIX [37](p) & 8.476 & 51.28 & 1.052 & 0.135 & 9.302 & 0.0025 \\
\hline AV18 & 6.923 & 45.68 & 1.526 & 0.065 & 8.466 & 0.0081 \\
AV18 [37](r) & 6.925 & 45.69 & 1.530 & 0.065 & 8.467 & 0.0081 \\
AV18 [37](p) & 6.923 & 45.68 & 1.524 & 0.065 & 8.466 & 0.0081 \\
AV18+UIX & 7.748 & 50.21 & 1.239 & 0.132 & 9.249 & 0.0075 \\
AV18+UIX [37](r) & 7.750 & 50.21 & 1.242 & 0.132 & 9.248 & 0.0075 \\
AV18+UIX [37](p) & 7.746 & 50.21 & 1.235 & 0.132 & 9.248 & 0.0075 \\
\hline \hline
\end{tabular}

TABLE I. Absolute value of binding energy, expectation value of kinetic energy (both in $\mathrm{MeV}$ ), and probabilities of the wave function components (all in \%) for ${ }^{3} \mathrm{H}$ (top) and ${ }^{3} \mathrm{He}$ (bottom) nuclei calculated with AV18 and AV18+UIX force models. Results of Ref. 37] obtained using coordinatespace (r) and momentum-space (p) frameworks are listed as well.
[1] H. Witała, W. Glöckle, J. Golak, A. Nogga, H. Kamada, R. Skibiński, and J. Kuros-Zolnierczuk, Phys. Rev. C 63 , 024007 (2001).

[2] J. Kuros-Zolnierczuk, H. Witała, J. Golak, H. Kamada, A. Nogga, R. Skibiński, and W. Glöckle, Phys. Rev. C 66, 024004 (2002).

[3] A. Deltuva, R. Machleidt, and P. U. Sauer, Phys. Rev. C 68, 024005 (2003).

[4] P. Doleschall, Phys. Rev. C 69, 054001 (2004).

[5] S. Ishikawa, Phys. Rev. C 75, 061002(R) (2007).

[6] A. Kievsky, M. Viviani, and S. Rosati, Phys. Rev. C 64, $024002(2001)$.

[7] A. Kievsky, M. Viviani, and L. E. Marcucci, Phys. Rev. C 69, 014002 (2004).

[8] R. B. Wiringa, V. G. J. Stoks, and R. Schiavilla, Phys. Rev. C 51, 38 (1995).

[9] B. S. Pudliner, V. R. Pandharipande, J. Carlson, S. C. Pieper, and R. B. Wiringa, Phys. Rev. C 56, 1720 (1997).

[10] S. Ishikawa, Phys. Rev. C 80, 054002 (2009).

[11] A. Deltuva, A. C. Fonseca, and P. U. Sauer, Phys. Rev. C 71, 054005 (2005).

[12] A. Deltuva, A. C. Fonseca, and P. U. Sauer, Phys. Rev. Lett. 95, 092301 (2005).

[13] J. R. Taylor, Nuovo Cimento B 23, 313 (1974); M. D. Semon and J. R. Taylor, Nuovo Cimento A 26, 48 (1975).

[14] E. O. Alt and W. Sandhas, Phys. Rev. C 21, 1733 (1980).

[15] E. O. Alt, P. Grassberger, and W. Sandhas, Nucl. Phys. B2, 167 (1967).

[16] A. Deltuva, K. Chmielewski, and P. U. Sauer, Phys. Rev. C 67, 034001 (2003).

[17] A. Deltuva, Ph.D. thesis, University of Hannover, 2003, http://edok01.tib.uni-hannover.de/edoks/e01dh03/37445470138dff]. Lazauskas and J. Carbonell, Phys. Rev. C 70, 044002 [18] A. Deltuva, A. C. Fonseca, and P. U. Sauer, Phys. Rev. C (2004). 72, 054004 (2005).

[19] A. Deltuva, A. C. Fonseca, and P. U. Sauer, Annu. Rev. Nucl. Part. Sci. 58, 27 (2008).

[20] A. Deltuva, K. Chmielewski, and P. U. Sauer, Phys. Rev. C 67, 054004 (2003).

[21] A. Deltuva, A. C. Fonseca, and P. U. Sauer, Phys. Rev. C 73, 057001 (2006).

[22] H. Witała, R. Skibinski, J. Golak, and W. Glöckle, Eur. Phys. J. A 41, 369 (2009); 41, 385 (2009).

[23] K. Sagara, H. Oguri, S. Shimizu, K. Maeda, H. Nakamura, T. Nakashima, and S. Morinobu, Phys. Rev. C 50, 576 (1994); K. Sagara (private communication).

[24] A. Deltuva, A. C. Fonseca, A. Kievsky, S. Rosati, P. U. Sauer, and M. Viviani, Phys. Rev. C 71, 064003 (2005).

[25] K. Ermisch et al., Phys. Rev. C 71, 064004 (2005).

[26] K. Sekiguchi et al., Phys. Rev. C 65, 034003 (2002).

[27] J. Fujita and H. Miyazawa, Prog. Theor. Phys. 17, 360 (1957)

[28] R. Machleidt, Phys. Rev. C 63, 024001 (2001).

[29] G. Rauprich, S. Lemaitre, P. Niessen, K. R. Nyga, R. Reckenfelderbäumer, L. Sydow, H. Paetz gen. Schieck, H. Witała, and W. Glöckle, Nucl. Phys. A535, 313 (1991).

[30] J. Strate et al., Nucl. Phys. A501, 51 (1989).

[31] H. R. Setze et al, Phys. Rev. C 71, 034006 (2005).

[32] S. Kistryn et al., Phys. Rev. C 72, 044006 (2005).

[33] S. Kistryn et al., Phys. Lett. B 641, 23 (2006).

[34] E. Stephan et al., Eur. Phys. J. A (2009).

[35] R. Lazauskas, Ph.D. thesis, University Joseph Fourier, Grenoble, 2003, http://tel.ccsd.cnrs.fr/documents/archives0/00/00/41/78/.

[36] R. Lazauskas, Few-Body Syst. 46, 37 (2009).

[37] A. Nogga, A. Kievsky, H. Kamada, W. Glöckle, L. E. Marcucci, S. Rosati, and M. Viviani, Phys. Rev. C 67, 034004 (2003). 